Proceedings

\title{
European and American Options Valuation by Unsupervised Learning with Artificial Neural Networks ${ }^{\dagger}$
}

\author{
Beatriz Salvador $1, *\left(\mathbb{D}\right.$, Cornelis W. Oosterlee ${ }^{2,3}$ and Remco van der Meer ${ }^{2,3}$ \\ 1 Universidade da Coruña, 15001 A Coruña, Spain \\ 2 CWI-Centrum Wiskunde \& Informatica, 1098 Amsterdam, The Netherlands; C.W.Oosterlee@cwi.nl \\ (C.W.O.); remcovdmeer0@gmail.com (R.v.d.M.) \\ 3 DIAM, Delft University of Technology, 2628 Delft, The Netherlands \\ * Correspondence: beatriz.salvador@udc.es \\ + Presented at the 3rd XoveTIC Conference, A Coruña, Spain, 8-9 October 2020.
}

Published: 19 August 2020

check for updates

\begin{abstract}
Artificial neural networks (ANNs) have recently also been applied to solve partial differential equations (PDEs). In this work, the classical problem of pricing European and American financial options, based on the corresponding PDE formulations, is studied. Instead of using numerical techniques based on finite element or difference methods, we address the problem using ANNs in the context of unsupervised learning. As a result, the ANN learns the option values for all possible underlying stock values at future time points, based on the minimization of a suitable loss function. For the European option, we solve the linear Black-Scholes equation, whereas for the American option, we solve the linear complementarity problem formulation.
\end{abstract}

Keywords: (non)linear PDEs; Black-Scholes model; artificial neural network; loss function; multi-asset options

\section{Introduction}

The interest in machine learning techniques, due to the remarkable successes in different application areas, is growing exponentially. ANNs are learning systems based on a collection of artificial neurons that constitute a connected network. Such systems "learn" to perform tasks, generally without being programmed with task-specific rules. Many different financial problems have also been addressed with machine learning. The financial application on which we focus is the valuation of financial derivatives with PDEs. Generally, we can distinguish between supervised and unsupervised machine learning techniques. The goal of the current work is to solve the financial PDEs by applying unsupervised machine learning techniques. In such a case, only the inputs of the network are known, and based on a suitable loss function that needs to be minimized, the ANN should "converge" to the solution of the PDE problem. We will price European and American options modeled by the Black-Scholes PDE and look for solutions for all future time points and stock values. Thus, linear and nonlinear partial differential equations need to be solved. 


\section{Artificial Neural Networks Solving PDEs}

We introduce the methodology following [1] to solve linear and nonlinear time-dependent PDEs by ANNs. Then, we write a general PDE problem as follows:

$$
\begin{array}{rl}
\mathcal{N}_{I}(v(t, x))=0, & x \in \widetilde{\Omega}, t \in[0, T], \\
\mathcal{N}_{B}(v(t, x))=0 & \text { on } \partial \widetilde{\Omega}, \\
\mathcal{N}_{0}\left(v\left(t^{*}, x\right)\right)=0 & x \in \widetilde{\Omega} \text { and } t^{*}=0 \text { or } t^{*}=T,
\end{array}
$$

where $v(t, x)$ denotes the solution of the $\operatorname{PDE}, \mathcal{N}_{I}(\cdot)$ is a linear or nonlinear time-dependent differential operator, $\mathcal{N}_{B}(\cdot)$ is a boundary operator, $\mathcal{N}_{0}(\cdot)$ is an initial or final time operator, $\widetilde{\Omega}$ is a subset of $\mathbb{R}^{D}$, and $\partial \widetilde{\Omega}$ denotes the boundary on the domain $\widetilde{\Omega}$. The goal is to obtain $\hat{v}(t, x)$ by minimizing a suitable loss function $L(v)$ over the space of $k$-times differentiable functions, where $k$ depends on the order of the derivatives in the PDE, i.e.,

$$
\arg \min _{v \in \mathcal{C}^{k}} L(v)=\hat{v}
$$

where we denote by $\hat{v}(t, x)$ the true solution of the PDE. A general expression for the loss function, defined in terms of the $L^{p}$ norm, including a weighting, is defined as follows [1,2]:

$$
L(v)=\lambda \int_{\Omega}\left|\mathcal{N}_{I}(v(t, x))\right|^{p} d \Omega+(1-\lambda) \int_{\partial \Omega}\left(\left|\mathcal{N}_{B}(v(t, x))\right|^{p}+\left|\mathcal{N}_{0}(v(t, x))\right|^{p}\right) d \gamma,
$$

where $\Omega=\widetilde{\Omega} \times[0, T]$ and $\partial \Omega$ the boundary of $\Omega$. Financial options with early-exercise features give rise to free boundary PDE problems. We will focus on the reformulation of the free boundary problem as a LCP. The generic LCP formulation reads:

$$
\begin{array}{rlrl}
\max \left(\mathcal{N}_{0}(v(t, x)), \mathcal{N}_{I}(v(t, x))\right) & =0, & & x \in \widetilde{\Omega}, t \in[0, T], \\
\mathcal{N}_{B}(v(t, x))=0, & & \text { on } \partial \widetilde{\Omega}, \\
\mathcal{N}_{0}\left(v\left(t^{*}, x\right)\right) & =0, & & x \in \widetilde{\Omega} \text { and } t^{*}=0 \text { or } t^{*}=T .
\end{array}
$$

Our expression for the loss function, to solve the linear complementarity problem, is as follows:

$$
L(v)=\lambda \int_{\Omega}\left|\max \left(\mathcal{N}_{0}(t, x, v), \mathcal{N}_{I}(t, x, v)\right)\right|^{p} d \Omega+(1-\lambda) \int_{\partial \Omega}\left(\left|\mathcal{N}_{B}(t, x, v)\right|^{p}+\left|\mathcal{N}_{0}(t, x, v)\right|^{p}\right) d \gamma .
$$

\section{Financial Derivatives Pricing PDEs}

In this section, the option pricing partial differential equation problems are presented. We briefly introduce the European asset model, the American and multi-asset options model being easily extended.

The underlying asset $S_{t}$ is assumed to pay a constant dividend yield $\delta$, and follows the geometric Brownian motion:

$$
d S_{t}=(\mu-\delta) S_{t} d t+\sigma S_{t} d W_{t}^{P}
$$

where $W_{t}^{P}$ is a Brownian motion. Assuming there are no arbitrage opportunities, the European option value follows from the Black-Scholes equation:

$$
\left\{\begin{array}{l}
\mathcal{L}(v)=\partial_{t} v+\mathcal{A} v-r v=0 \quad S \in \widetilde{\Omega} t \in[0, T) \\
v(T, S)=H(S)
\end{array}\right.
$$


where operator $\mathcal{A}$ is the classical Black-Scholes operator, and function $H$ denotes the option's payoff. Then, the loss function is defined as:

$$
\begin{aligned}
L(v) & =\lambda \int_{\Omega}|\mathcal{L}(v(t, x))|^{p} d \Omega \\
& +(1-\lambda) \int_{\partial \Omega}\left(|v(t, x)-G(t, x)|^{p}+|v(t, x)-H(x)|^{p}\right) d \gamma,
\end{aligned}
$$

where functions $G$ and $H$ denote the values of the spatial boundary conditions and final condition, respectively. The integral terms in the loss function are approximated by Monte Carlo techniques.

\section{ANN Option Pricing Results}

We start with a European put option, with the following parameters' values: $\sigma=0.25, r=0.04$, $T=1, K=15, S_{\infty}=4 K, \delta=0.0$. In Figure 1, the ANN-based, trained, and the analytical solution are plotted for two time instances. The relative error, with $\lambda=0.5$, is equal to $2.23 \times 10^{-4}$.
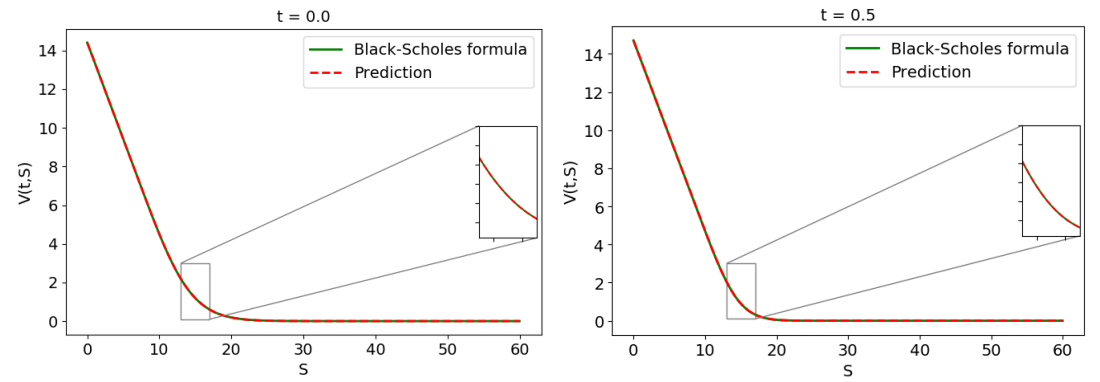

Figure 1. European put option for different times instances, $t=0, t=0.5$, with $\lambda=0.5$.

Finally, in order to show the accuracy of the method applied to train the ANN to price American options depending on two asset prices, the relative error is presented in Table 1.

Table 1. Error for different multi-asset American options.

\begin{tabular}{cc}
\hline & Error \\
\hline Max-call & $1.73 \times 10^{-3}$ \\
Spread & $2.45 \times 10^{-3}$ \\
Arithmetic average put & $6.42 \times 10^{-3}$ \\
\hline
\end{tabular}

Author Contributions: Investigation and writing-original draft preparation: B.S., Supervision: C.W.O., validation: R.v.d.M. All authors have read and agreed to the published version of the manuscript.

Funding: This research was funded by ERCIM fellowship.

Conflicts of Interest: The authors declare no conflict of interest.

\section{References}

1. van der Meer, R.; Oosterlee, C.; Borovykh, A.T. Optimally weighted loss function for solving PDEs with neural Networks. arXiv 2020, arXiv:2002.06269.

2. Raissi, M.; Karniadakis, G.E.; Perdikris, P. Physics informed deep learning (part I): Data-driven solutions of nonlinear partial differential equations. arXiv 2017, arXiv:1711.10561v1.

(c) 2020 by the authors. Licensee MDPI, Basel, Switzerland. This article is an open access article distributed under the terms and conditions of the Creative Commons Attribution (CC BY) license (http:/ / creativecommons.org/licenses/by/4.0/). 